\title{
The Kager's fat pad radiological anatomy revised
}

\author{
Paweł Szaro ${ }^{1,2}$ - Mateusz Polaczek ${ }^{3} \cdot$ Bogdan Ciszek $^{4}$
}

Received: 26 June 2020 / Accepted: 12 August 2020 / Published online: 19 August 2020

(c) The Author(s) 2020

\begin{abstract}
Purpose The aim of the study was to map connections within the Kager's fat pad between the structures which limit it. Methods A retrospective re-review of 200 ankle magnetic resonance imaging (MRI) examination was conducted. Connections within the Kager's fat pad between the superior peroneal retinaculum, the fibulotalocalcaneal ligament, the posterior talocalcaneal ligament, the flexor hallucis longus, the paratenon of the Achilles tendon, the flexor retinaculum and bones were studied and a model of the connections was constructed.

Results The superior peroneal retinaculum was directly connected with the fibulotalocalcaneal ligament in $85.5 \%$ of cases, the lateral part of the paratenon in $82.5 \%$, the processus posterior tali in $78.5 \%$, the posterior talofibular ligament in $32 \%$, the flexor retinaculum in $29.5 \%$ and the anterior talofibular ligament in $9 \%$.

The fibulotalocalcaneal ligament was connected with the paratenon (on the medial side $88.5 \%$, on the lateral side $68.5 \%$ ), the flexor retinaculum in $70 \%$, the posterior process of the talus in $79 \%$, the osteofibrosus tunnel for the flexor hallucis longus in $53 \%$, the posterior talofibular ligament in $43.5 \%$ and the calcaneofibular ligament in $10.5 \%$.

The posterior talocalcaneal ligament was connected with the fibulotalocalcaneal ligament in $71 \%$, with the osteofibrosus tunnel for the flexor hallucis longus in $76.5 \%$, with the flexor retinaculum in $70 \%$. The plantaris tendon showed projection to the crural fascia in 34 of $\%$ cases.

Conclusion In the Kager's fat pad there are present more connections than previously reported. All the connections unit at the level of the posterior process of the talus.
\end{abstract}

Keywords Kager fat pad · Achilles tendon $\cdot$ Retrocalcaneal bursa $\cdot$ Crural fascia

\section{Abbreviations}

ATFL The anterior talofibular ligament

CFL Calcaneofibular ligament

FHL The flexor hallucis longus

FOV Field of view

FSE Fast spin-echo

FTCL The fibulotalocalcaneal ligament
KFP The Kager's fat pad

MRI Magnetic resonance imaging

PD Proton density

PTCL The posterior talocalcaneal ligament

PTFL The posterior talofibular ligament

TE Echo time
Paweł Szaro

pawel.szaro@gu.se

1 Department of Radiology, Institute of Clinical Sciences, Sahlgrenska Academy, University of Gothenburg, Göteborgsvägen 31, 43180 Gothenburg, Sweden

2 Department of Musculoskeletal Radiology, Sahlgrenska University Hospital, Göteborgsvägen 31, 43180 Gothenburg, Sweden

3 Third Department of Lung Diseases and Oncology, National Tuberculosis and Lung Diseases Research Institute, Plocka 26, 01138 Warsaw, Poland

4 Department of Descriptive and Clinical Anatomy, Medical University of Warsaw, Warsaw, Poland

\section{Introduction}

Connections within the Kager's fat pad (KFP) have not been comprehensively studied. The KFP is an adipose structure located between the Achilles tendon, the flexor hallucis longus (FHL) and the calcaneus [8, 28]. Due to the KFP's close relation to the Achilles tendon, it can be involved in traumatic changes in the tendon's midportion as well as the entheses or other conditions like a tumor or infection [11, $22,26]$, but the connections can provide stability [5, 10]. The relatively large size of the KFP enables its individual parts to have different functions and exhibit slightly different 
mechanical functions and a slightly different sort of pathology $[3,15,28]$. The wedge-shaped part of the KFP participates in the lubrication of the anterior outline and distal part of the Achilles tendon and evenly distributes stress at the Achilles enthesis, and removes debris from the retrocalcaneal bursa [2, 22]. The KFP also has fibrous bands stretching between the surrounding structures in a diversified distribution. Different parts of the KFP may play different functions. In previous studies, only two connections have been found within the KFP: the posterior talocalcaneal ligament (PTCL) and the fibulotalocalcaneal ligament (FTCL) [6, 19]. The PTCL originates on the lateral tubercle on the posterior process of the talus and inserts on the superior outline of the calcaneus [6]. The FTCL originates in the anterior part of the malleolar groove, runs medially, then divides into flat sheet-like laminas, the superomedial and the inferolateral. The first one inserts onto the lateral tubercle while the second one inserts onto the superior outline of the calcaneus [19]. It should be noted that there is an inconsequence in the nomenclature of the FTCL's laminas. The one which inserts onto talus is sometimes called horizontal or talar, while the one which runs toward the calcaneus, inferolateral or peroneocalcaneal [20].

The plantaris tendon may have an insertion into the deep crural fascia which corresponds with type 4 according to Olewik et al. (2016) [18]. It was previously mentioned that the plantaris tendon may have a connection to the flexor retinaculum or to the crural fascia [1,27].

MRI is an excellent method that allows tissue differentiation with high resolution $[3,7]$. Thanks to this, the presence of even fine fiber bands within the KFP can be precisely demonstrated [21,23].

Despite the common occurrence of soft tissue alterations in the KFP [3], to our knowledge, there is a lack of modern radiological research on a large amount of material describing and classifying the presence of fibrous connections and ligaments in the KFP.

The purpose of this study was to examine the occurrence and variability of bands and ligaments in the KFP and construct a map of the connections based on MRI.

\section{Materials and methods}

We conducted a retrospective re-evaluation of 200 ankle MRI of athletes (112 males and 88 females; age range 16-47 years, mean 29 years; 101 right ankles and 99 left ones). Exclusion criteria were the presence of the os trigonum ( 21 cases), a history of previous fracture and obvious abnormality within the KFP because of the possibility of a focal reaction and finally remaining orthopedic hardware due to the possible artifacts ( 16 cases). Totally 200 patients with ankle MRI fulfilling inclusion and exclusion criteria were included in our study. MRI examinations were performed at our institution over six months (between November 2018 and April 2019), about 2-4 weeks after the ankle injury (mean duration 2.8 weeks) for clinical purposes. All examinations were performed using MRI machine Ingenia 3.0 T MR system (Philips Healthcare) with a dedicated ankle coil.

To obtain the best possible differentiation of fat and fibrous tissue, we used sequences without fat tissue saturation in PD (proton density) and T2-weighted images in three different planes. The sagittal and axial FSE (fast spin-echo) sequences had a field of view (FOV) of $14 \times 14 \mathrm{~cm}$, a slice thickness of $3 \mathrm{~mm}$ without spacing. The coronal sequences had a FOV of $10 \times 8 \mathrm{~cm}$. Matrix in axial plane $256 \times 218$, in coronal plane $256 \times 230$ in sagittal plane $320 \times 272$. The echo time in PD 20 while in T2 FSE $4000 \mathrm{~ms}$.

In the study, we studied connections between the following structures: the peroneal tendons and superior peroneal retinaculum, the flexor hallucis longus, the paratenon, the medial vascular space, the tibialis posterior and the flexor digitorum longus. The hypothesis of our study was that located within the KFP are fascia projections joining the different limitations of the KFP.

After coding the results in the table, a model of connections developed in the KFP at the level of the posterior process of the talus was made. Due to earlier reports regarding the plantaris tendon and its relationship to the fascia, its variant according to Olewik et al. [18] was applied.

\section{Results}

In the KFP a lot more connections than only the FTCL or the posterior talofibular ligament (PTFL) can be found as was reported in previous studies. Most of the connections we have found are projections of the crural fascia orientated in the frontal plane, therefore their assessment in the horizontal plane is the most optimal. Due to the very narrow anterior-posterior diameter, assessment in the frontal plane is more difficult. The sagittal plane helps to assess the cranio-caudal distension of the described connections.

All connections could be identified at the level of the posterior process of the talus on the axial plane. The PTCL was identified in $n=161(80.5 \%)$ (Figs. 1, 2, 6, Table 1). This ligament originates from the lateral tubercle of the posterior process of the talus where it connects with the superomedial lamina of the FTCL and then descends to the superior outline of the calcaneal tuber (Fig. 6). In $n=153$ (76.5\%) of cases, its vertical projection was connected directly to the FHL (Figs. 2c, 6), and indirectly via the FTCL in 39 cases (19.5\%) (Fig. 2b-d).

The superior peroneal retinaculum's connections were observed to be variable (Fig. 10, Table 1). The connections most often seen were with the FTCL, the lateral part of the 


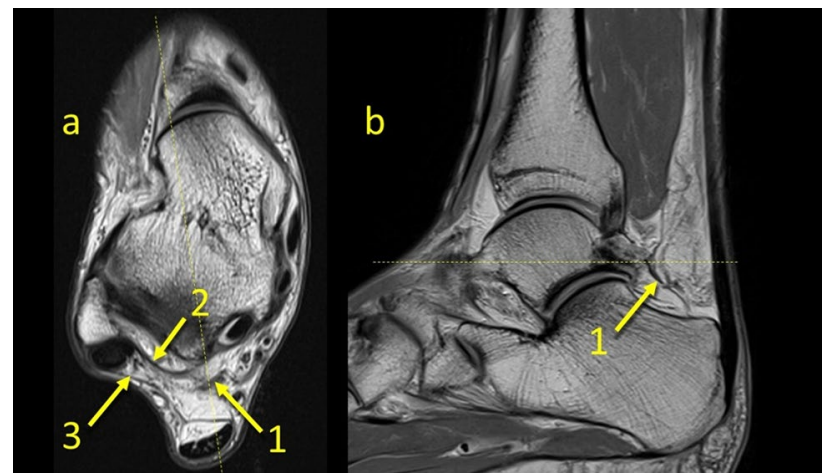

Fig. 1 The posterior talocalcaneal ligament. 1 - the FTCL, 2 - the superomedial lamina of the FTCL, $\mathbf{3}$ - the superior peroneal retinaculum

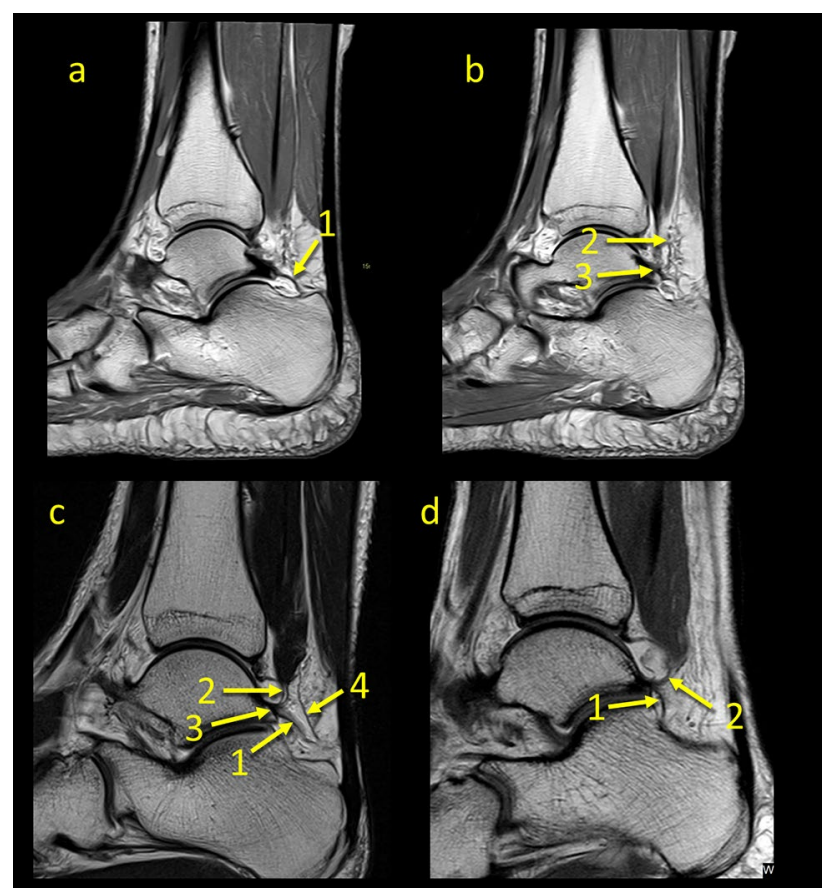

Fig. 2 The posterior talocalcaneal ligaments connections in two different patients, a and b, c and d. 1 - the PTCL, 2 - the superomedial lamina of the FTCL connecting the FHL, $\mathbf{3}$ - the superomedial lamina of the FTCL's insertion on the posterior process of the talus, 4 - the communication between the PTCL and the FHL

paratenon of the Achilles tendon and the posterior process of the talus (Table 1, Fig. 2). Connections with the lateral ankle ligament complex or the flexor retinaculum are visible but much less so.

In $n=18$ cases (9\%), communication to the anterior talofibular ligament (ATFL) was found as prolongation or extension of the superior peroneal retinaculum fibers over the external outline of the lateral malleolus to the superior part of the ATFL (Figs. 3, 4, 5, 6).
The FTCL is a structure which is located between the lateral malleolus and the medial vascular space at the level of the posterior process of the talus. It was present in all cases in two variants either as two laminas, type $1(n=158,79 \%)$ or as a single structure, type $2(n=42$, $21 \%$ ), Fig. 7. Its origin is located in the fossa malleoli lateralis, then runs medially where in type 1 it branches into the superomedial and inferolateral lamina. In type 2, the superomedial lamina is not present. This ligament, thanks to its central localization and connections, serves as a "common intersection points" between the structures which limit the KFP (Table 1). In some cases, $n=22$ $(11 \%)$, division of the FTCL is already seen at its origin on the fibula, making the superomedial and inferolateral laminas longer than usual (Fig. 7c). The superomedial lamina inserts into the lateral tubercle of the processus posterior tali, contributing to the osseofibrous tunnel for the FHL, while the inferolateral lamina which runs inferiorly and inserts onto the calcaneus.

The most constant FTCL connection was with the paratenon of the Achilles tendon. The connection with the flexor retinaculum was the most medially located connection, while that with the FHL is the most superior connection (Table 1).

At FTCL's origin in the fossa malleoli lateralis, the connection with the PTFL was seen in $n=87$ cases $(43.5 \%)$ (Fig. 5), while the connection with the calcaneofibular ligament (CFL) was identified in $n=21$ cases (10.5\%) (Table 1). In no cases was direct contact with the AFTL recognized. We noticed the FTCL's connections to the FHL in 105 cases $52.5 \%$ of cases (Fig. 2).

The plantaris tendon in type 4 according to Olewnik et al. [18], $n=67$ (34\%), showed a projection of its insertion to the crural fascia. The plantaris tendon was located medially to the Achilles tendon in direct contact with the medial part of the paratenon. The accessory slip from the flexor retinaculum with the medial part of the paratenon was noticed in $n=77$ cases (38.5\%). We found one case of the peroneus quartus tendon but the connections within the KFP did not differ from other cases. No variations of the solus tendon were identified.

The paratenon of the Achilles tendon was connected anteriorly, medially and laterally with the FTCL (Table 1) and with the superior peroneal retinaculum. Anteriorly and medially connection to the node-like structure can be seen.

The lateral tubercle of the posterior process of the talus is the site of insertion of the PTCL, the superomedial lamina of the FTCL and part of the PTFL. The prolongation of the superomedial lamina of the FTCL continues to the medial tubercle, limiting the osteofibrous tunnel for the FHL, and finally joins with the flexor retinaculum. Often the septum between the flexor digitorum longus and the tibialis posterior tendon is visible ( $n=157$ cases, $79 \%)$. 
Table 1 Number of direct connections between the structures include in the study, \% in brackets, $n=200(100 \%)$

\begin{tabular}{|c|c|c|c|c|c|c|c|c|c|c|c|}
\hline & SPR & ATFL & CFL & PTFL & FTCL & PTCL & PAT med & PAT lat & PPT & OF-FHL & FR \\
\hline SPR & $\mathrm{x}$ & $18(9)$ & $0(0)$ & $64(32)$ & $171(85.5)$ & $17(8.5)$ & $39(19.5)$ & $165(82.5)$ & $157(78.5)$ & $0(0)$ & $59(29.5)$ \\
\hline ATFL & & $\mathrm{x}$ & ns & ns & $0(0)$ & $0(0)$ & $0(0)$ & $0(0)$ & $0(0)$ & $0(0)$ & $0(0)$ \\
\hline CFL & & & $\mathrm{x}$ & ns & $21(10.5)$ & $0(0)$ & $0(0)$ & $0(0)$ & $0(0)$ & $0(0)$ & $0(0)$ \\
\hline PTFL & & & & $\mathrm{x}$ & $87(43.5)$ & $0(0)$ & $0(0)$ & $0(0)$ & ins & $0(0)$ & $0(0)$ \\
\hline FTCL & & & & & $\mathrm{x}$ & $\begin{array}{l}142(71)^{*} \\
133 \\
(66.5)^{* *}\end{array}$ & 177 (88.5) & 137 (68.5) & $158(79) * * *$ & $105(52.5)$ & $140(70)$ \\
\hline PTCL & & & & & & $\mathrm{x}$ & $16(8)$ & $24(12)$ & orig & $153(76.5)$ & $36(18)$ \\
\hline PAR med & & & & & & & $\mathrm{x}$ & $* * * *$ & $0(0)$ & $0(0)$ & $117(58.5)$ \\
\hline PAR lat & & & & & & & & $\mathrm{x}$ & $0(0)$ & $0(0)$ & $0(0)$ \\
\hline PPT & & & & & & & & & $\mathrm{x}$ & ins & $0(0)$ \\
\hline FHL & & & & & & & & & & $\mathrm{x}$ & $0(0)$ \\
\hline FR & & & & & & & & & & & $X$ \\
\hline
\end{tabular}

ins. insertion, $F R$ the flexor retinaculum, $n s$ not studied, $O F-F H L$ osteofibrous tunnel for FHL, orig.-origin, $S P R$ the superior peroneal retinaculum, PAT med the medial part of the paratenon, PAT lat. the lateral part of the paratenon,, PPT the posterior process of talus

*The superomedial lamina of FTCL

**Inferolateral lamina of the FTCL

*** Only variant one of the FTCL

****Anatomical continuity

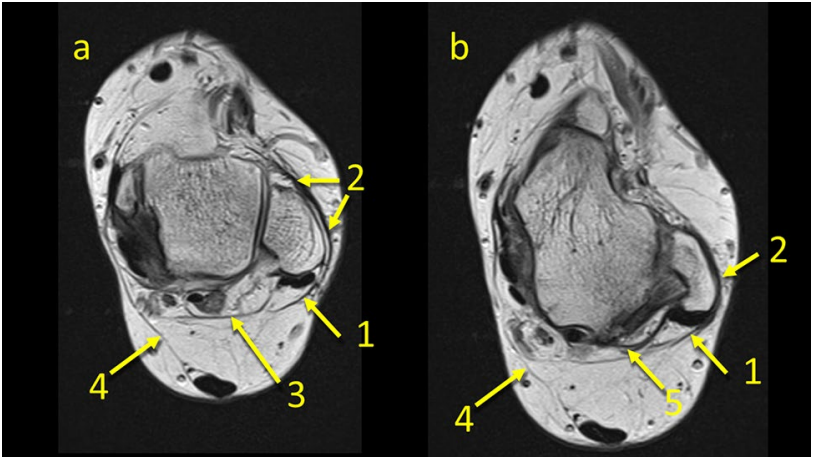

Fig. 3 The communication between the ATFL and the superior peroneal retinaculum. $\mathbf{1}$ - the superior peroneal retinaculum, $\mathbf{2}$ - the fibers connecting the superior peroneal retinaculum with the ATFL, 3 - the FTCL, 4 - the communication between the flexor retinaculum and the medial paratenon, $\mathbf{5}$ - the superomedial lamina of the FTCL

\section{Discussion}

In our study, we found many more connections than the two previously described ligaments. To our knowledge, there are no radiological studies regarding all connections within the KFP. In some earlier published studies only on cadavers, only information about the posterior talocalcaneal ligament and the FTCL can be found [13, 20]. However, when assessing ankle MRI, many more connections within the KFP can be observed. Our study revealed

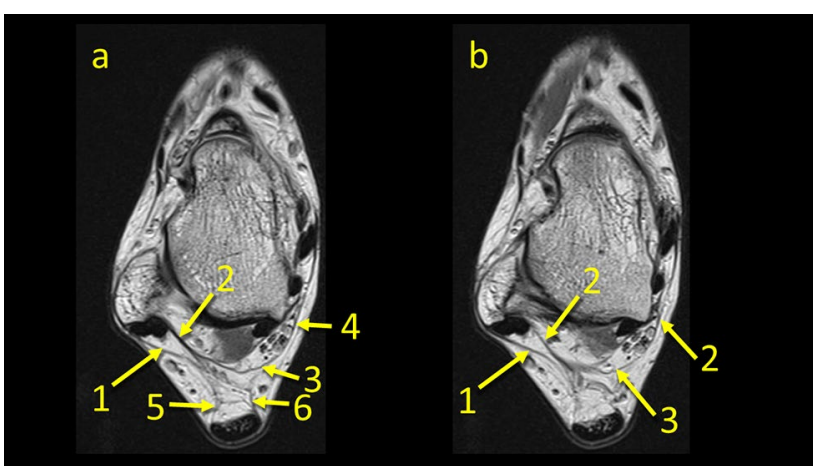

Fig. 4 The communication between the superior peroneal retinaculum and the flexor retinaculum. $\mathbf{1}$ - the superior peroneal retinaculum, $\mathbf{2}$ - the FTCL, $\mathbf{3}$ - the communication between the superior peroneal retinaculum and the flexor retinaculum via the FTCL, $\mathbf{4}$ - the flexor retinaculum, $\mathbf{5}$ - the medial part of paratenon, $\mathbf{6}$ - the lateral part of paratenon

the presence of variable connections and some "common intersection points" between the fascial projections or ligaments within the KFP. The most classic example is the occurrence of a node-like structure located in the central part of the KFP at the level of the posterior process of the talus, as part of the FTCL. Due to the small amount of research, unambiguous nomenclature for fascial connections is missing $[19,20]$. The connections revealed in our study are part of the fascial system proposed previously [25], but because of the complexity of further development in fascial nomenclature is required. 


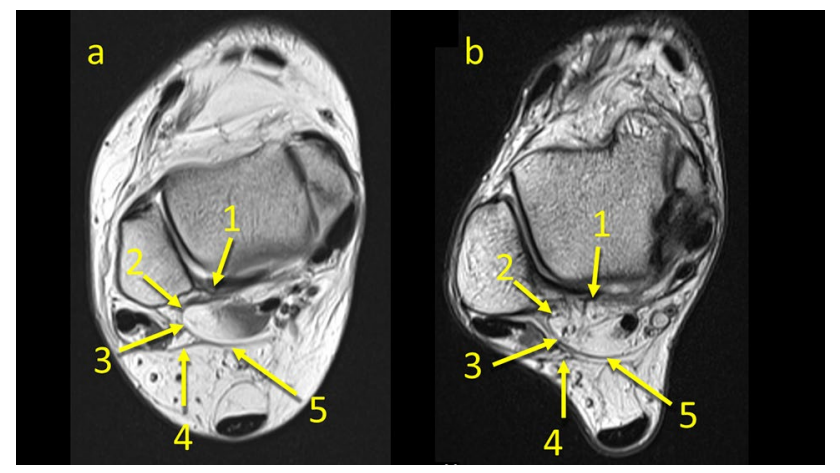

Fig. 5 Two patients with communication between the FTCL and the PTFL (the arrow number two). 1 - the PTFL, 2 - the communication, $\mathbf{3}$ - the FTCL, $\mathbf{4}$ - the superior peroneal retinaculum, $\mathbf{5}$ - the superomedial lamina of the FTCL
The occurrence of such connecting structures confirms the theories of occurrence of the intermuscular myofascial connections which can influence muscle and tendon functions [12]. A fascia may have passive transmitting mechanical forces generated by skeletal muscle. Our study revealed the crural fascia connections to tendons, e.g., to the FHL or to the paratenon. The plantaris tendon's connection to the crural fascia and its projections to the paratenon may partially explain the anatomical basis of the pathology of Achilles tendon tendinopathy and paratenonitis [18]. Some authors reveal that the connection of the KFP to the Achilles tendon helps to maintain its stability $[5,10]$. The connections of the KFP with the paratenon revealed in our study can be an anatomical background which may decrease the mutual glide of the paratenon and the Achilles tendon. This reduction can develop of the overuse changes, the paratendinitis or the tendinopathy of the Achilles tendon.
Fig. 6 The node-like structure between ligaments. 1 - the node-like structure, $\mathbf{2}$ - the PTCL, 3 - the FTCL, 4 - the superomedial lamina of the FTCL, $\mathbf{5}$ - the connection with the FHL, $\mathbf{6}$ - the connection to the flexor retinaculum
Fig. 7 Three variants of the FTCL. 1 - the FTCL, 2 - the superomedial lamina of the FTCL, $\mathbf{3}$ - the communication between the superior peroneal retinaculum and the flexor retinaculum via the FTCL, 4 - the lateral part of the paratenon, 5 the superior peroneal retinaculum, $\mathbf{6}$ - the inferolateral lamina of the FTCL, 7 - the "nodelike" structure
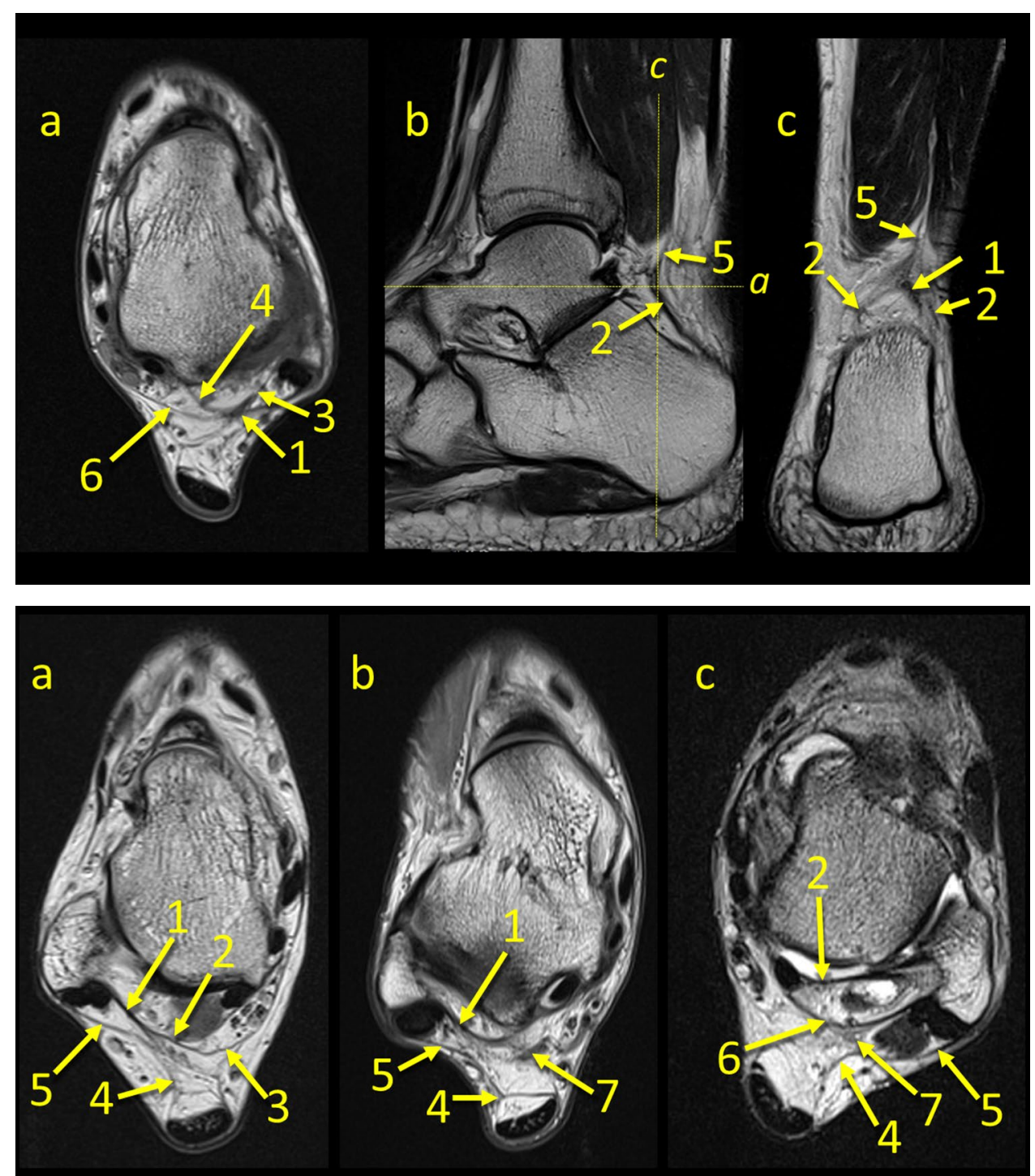
The presence of connections to ligaments such as the PTFL or CFL helps to understand why isolated tears are rare in the ankle area $[9,17]$. Given the existence of the connections within the KFP, it is somewhat more logical to understand the coexistence of the overuse of the Achilles tendon with alterations in other structures in the ankle. We believe, therefore, that injuries to the ankle area should be considered holistically, taking into account the mechanism of the injury and not just one individual injured structure.

Several connections revealed in the posterior ankle area look like the connections described earlier in the lateral ankle complex [9, 17, 30]. Furthermore, there are some similarities between the KFP and the Hoffa fat pad [14]. Alterations in these structures result in the alteration of the KFP's structure and obscure its outline on radiological imaging which is known as the Kager's fad pad sign [10]. Impingement, inflammation, overuse or infection in the KFP may finally lead to the lipomatous tissue necrosis [4, 22, 29]. The KFP shows alterations in the case of the Haglund deformity because of the impingement and fat edema, synovitis in the deep calcaneal bursa and a high signal in the Achilles tendon insertion $[3,16,30]$. However, no connections were revealed in this part of the KFP which allows it to move freely in the retrocalcaneal bursa $[8,28]$. We revealed that the KFP is connected with paratenon on both the medial and lateral sides which may explain the KFP's involvement in the pathology of the enthesis organ of the Achilles tenon or paratenon [22, 24, 26].

The posterior process of the talus is a point of insertion of the superomedial lamina of the FTCL which is a connection between the lateral side of the ankle by means of the PTFL $[19,20]$. This is the reason why we decided to evaluate the KFP's connections at this level. Medially, the FTCL runs and connects with the flexor retinaculum. Inferiorly, it connects with the posterior talocalcaneal ligament. Previously published results indicate that the inferolateral lamina inserts on the lateral part of the tuber calcanei, but in our study, we found that this arch-shaped structure inserts on the superior surface of the calcaneus (Fig. 6c). The discrepancy may be due to the fact that previous work was based on scarce material, for example Peduto et al. dissected six cadavers [20] and Pastore et al. dissected ten cadavers [19] (Fig. 8, 9, 10).

Our study showed that the PTCL was found in about $70 \%$ which is similar to number reported by Pastore et al. [19], while a recently published study by Iovane et al. [6] indicate that the incidence of the PTCL is much lower, estimated at nearly $9 \%$. It is difficult to determine where these discrepancies come from, possibly a slightly different research methodology or a different selection of material.

In some cases, the plantaris tendon attaches to the fascial structures at the site where the flexor retinaculum meets the medial part of the paratenon of the Achilles tendon. Under adverse conditions, the plantaris tendon may

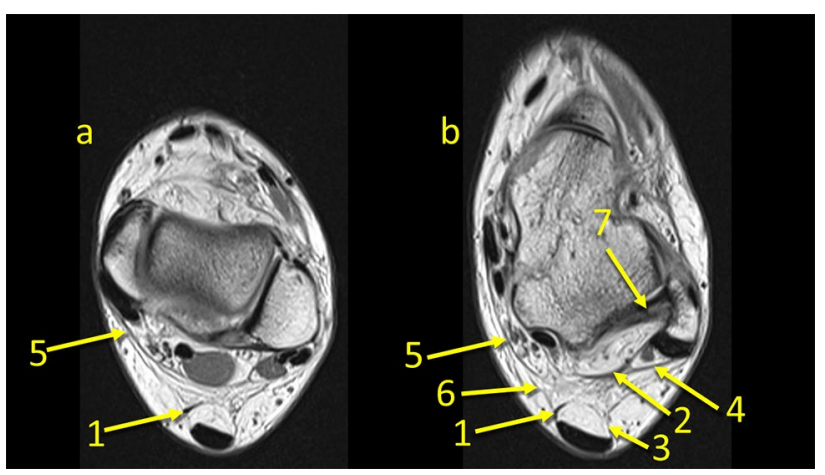

Fig. 8 The connection between the paratenon and the superior peroneal retinaculum and the flexor retinaculum. 1 - the plantaris tendon, $\mathbf{2}$ - the FTCL, $\mathbf{3}$ - the lateral part of paratenon, $\mathbf{4}$ - the superior peroneal retinaculum, $\mathbf{5}$ - the flexor retinaculum, $\mathbf{6}$ - the connection between the paratenon and the flexor retinaculum, 7 - the PTFL

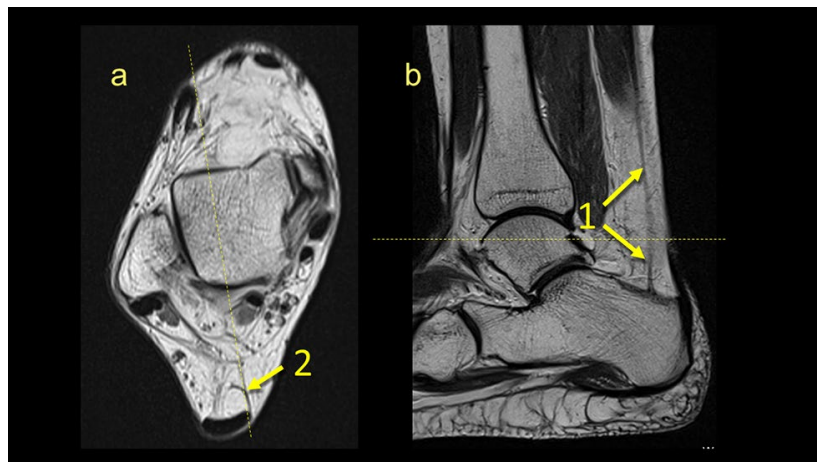

Fig. 9 The connection between the plantaris tendon and the paratenon. $\mathbf{1}$ - the plantaris tenon, $\mathbf{2}$ - the plantaris inserts into the medial part of the paratenon

have an important role in triggering Achilles tendonitis or overuse [18]. As in the case of the palmaris longus tendon connecting with the palmar fascia, the plantaris tendon connects with the crural fascia and may have a connection with the plantar fascia. This fascial connection was previously described but in our study.

We reveal that this connection extends further to other structures making the KFP a connecting, not a separating structure. Considering previously published results of functional studies approach to the KFP's function should be revised [15].

There are some limitations to this study. Due to its retrospective character, we had no influence on the thickness of the layers in the MRI examination because the examinations were performed because of clinical indications, in a defined protocol. Another limitation is the lack of surgical-anatomical correlation and the presence of discrete degenerative changes. 

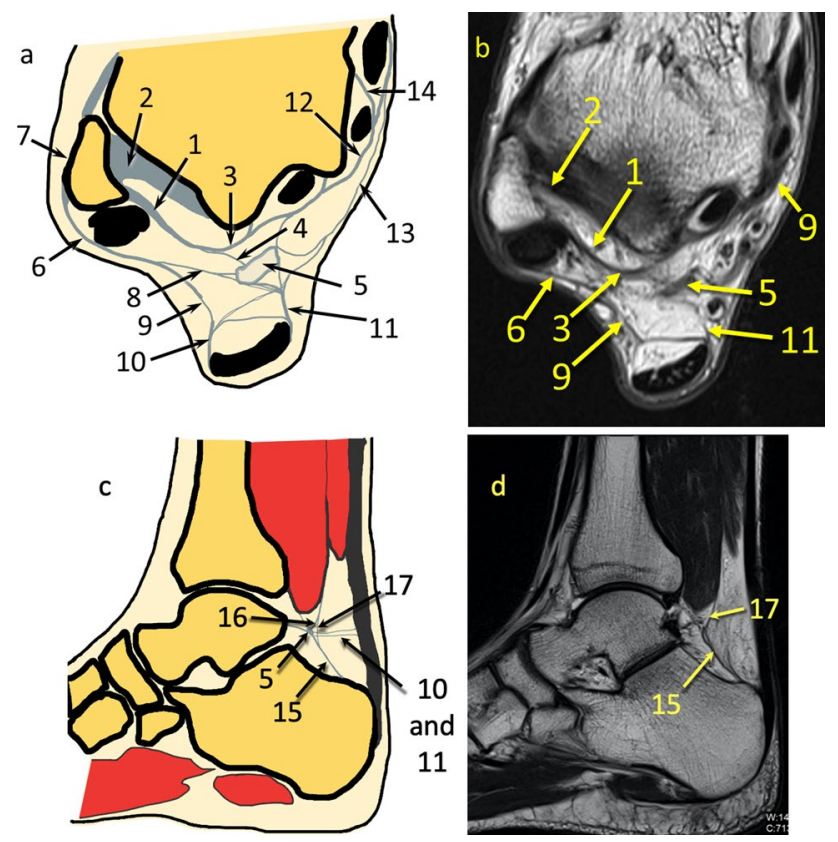

Fig. 10 Communications within the KFP at the level of the posterior process of the talus. a and c- general map of the communications between different fibrotic structures, $\mathbf{b}$ and $\mathbf{d}$ - example of the patient. $\mathbf{1}$ - the FTCL, 2- the PTFL, 3- the superomedial lamina of the TFCL, 4- branch of the TFCL to the node-like structure, 5- the node-like structures, 6- the superior peroneal retinaculum, 7- connection between the ATFL and the superior peroneal retinaculum, 8connection between the node-like structure and the superior peroneal retinaculum, 9- connection between the lateral part of the paratenon (10) and the superior peroneal retinaculum, 11- connection between the node-like structure and the medial part of the retinaculum, 12- the flexor retinaculum, 13- connection between the node-like structure and the flexor retinaculum, 14- the septa between the flexor digitorum longus and the tibialis posterior, $\mathbf{1 5}$ - the PTCL, $\mathbf{1 6}$ - connection between the PTCL and FHL via the node-like structure, 17 - direct connection between the PTCL and FHL

\section{Conclusion}

There are significantly more connections in KFP than previously reported. The KFP is a structure where the crural fascia's projections from the KFP limiting's structures join. The most important "coordination structure" is located at the level of the posterior process of the talus where the following unite: the PTCL, the FTCL and its projections to the superior peroneal retinaculum, the flexor retinaculum joint, the paratenon of the Achilles tendon and the FHL. The connections shown in our study indicate the presence of a fascial integration system within the KFP. The current study revealed that the KFP can play an important role in the pathology of its limiting structures. One structure's dysfunction due to connections may cause the dysfunction of another. The existence of these connections may partly explain the multifactorial cause of Achilles tendinopathy.
Author contributions All authors contributed to the study conception and design. Material preparation, data collection and analysis were performed by all authors. The first draft of the manuscript was written by the first author and all authors commented on previous versions of the manuscript. All authors read and approved the final manuscript.

Funding Open access funding provided by University of Gothenburg.

\section{Compliance with ethical standards}

Conflicts of interest The authors declare that they have no conflict of interest.

Ethics approval Ethical approval was waived by the local Ethics Committee in view of the retrospective nature of the study and that all the procedures being performed were part of routine care. The Institutional Ethics Committee was informed about the ongoing retrospective study and the Committee stated there was no need for its approval.

Availability of data and material Data transparency: Yes.

Code availability Software application or custom code: Yes.

Consent to participate Not applicable. This research study was conducted retrospectively from data obtained for clinical purposes.

Consent for publication Not applicable. This research study was conducted retrospectively from data obtained for clinical purposes.

Open Access This article is licensed under a Creative Commons Attribution 4.0 International License, which permits use, sharing, adaptation, distribution and reproduction in any medium or format, as long as you give appropriate credit to the original author(s) and the source, provide a link to the Creative Commons licence, and indicate if changes were made. The images or other third party material in this article are included in the article's Creative Commons licence, unless indicated otherwise in a credit line to the material. If material is not included in the article's Creative Commons licence and your intended use is not permitted by statutory regulation or exceeds the permitted use, you will need to obtain permission directly from the copyright holder. To view a copy of this licence, visit http://creativecommons.org/licenses/by/4.0/.

\section{References}

1. Cummins EJ, Anson BJ (1946) The structure of the calcaneal tendon (of Achilles) in relation to orthopedic surgery, with additional observations on the plantaris muscle. Surg Gynecol Obstet 83:107-116

2. Ghazzawi A, Theobald P, Pugh N et al (2009) Quantifying the motion of Kager's fat pad. J Orthop Res 27:1457-1460. https:// doi.org/10.1002/jor.20900

3. Gheno R, Nico MAC, Buck FM et al (2010) Edematous processes within Kager fat pad: magnetic resonance imaging, gross anatomical, and histological studies in cadavers with clinical correlation. J Comput Assist Tomogr 34:621-625. https://doi.org/10.1097/ RCT.0b013e3181d275cc

4. Godoy-Santos AL, Bordalo-Rodrigues M, Rosemberg L et al (2014) Kager's fat pad inflammation associated with HIV infection and AIDS: MRI findings. Skeletal Radiol 43:1257-1262. https://doi.org/10.1007/s00256-014-1931-5 
5. He L, Genin J, Delzell P (2016) Ultrasound diagnosis and percutaneous treatment of Achilles tendon tethering: a case series. Skeletal Radiol 45:1293-1298. https://doi.org/10.1007/s0025 6-016-2416-5

6. Iovane A, Palma A, Messina G et al (2020) The posterior talocalcaneal ligament: an MRI evaluation. Surg Radiol Anat 42:11671174. https://doi.org/10.1007/s00276-020-02506-7

7. Juras V, Welsch G, Bär P et al (2012) Comparison of $3 \mathrm{~T}$ and $7 \mathrm{~T}$ MRI clinical sequences for ankle imaging. Eur J Radiol 81:18461850. https://doi.org/10.1016/j.ejrad.2011.05.023

8. Kachlik D, Baca V, Cepelik M et al (2008) Clinical anatomy of the retrocalcaneal bursa. Surg Radiol Anat 30:347-353. https:// doi.org/10.1007/s00276-008-0335-4

9. Kakegawa A, Mori Y, Tsuchiya A et al (2019) Independent attachment of lateral ankle ligaments: anterior talofibular and calcaneofibular ligaments - a cadaveric study. J Foot Ankle Surg 58:717-722. https://doi.org/10.1053/j.jfas.2018.12.009

10. Kinugasa R, Taniguchi K, Yamamura N et al (2018) A multimodality approach towards elucidation of the mechanism for human achilles tendon bending during passive ankle rotation. Sci Rep 8:4319. https://doi.org/10.1038/s41598-018-22661-7

11. Kokulu K, Akça HŞ (2017) The Kager's fat pad sign. J Emerg Med 53:760-761. https://doi.org/10.1016/j.jemermed.2017.08.014

12. Krause F, Wilke J, Vogt L, Banzer W (2016) Intermuscular force transmission along myofascial chains: a systematic review. J Anat 228:910-918. https://doi.org/10.1111/joa.12464

13. Ly JQ, Bui-Mansfield LT (2004) Anatomy of and abnormalities associated with Kager's fat Pad. AJR Am J Roentgenol 182:147154. https://doi.org/10.2214/ajr.182.1.1820147

14. Magi M, Branca A, Bucca C, Langerame V (1991) Hoffa disease. Ital J Orthop Traumatol 17:211-216

15. Malagelada F, Stephen J, Dalmau-Pastor M et al (2020) Pressure changes in the Kager fat pad at the extremes of ankle motion suggest a potential role in Achilles tendinopathy. Knee Surg Sports Traumatol Arthrosc 28:148-154. https://doi.org/10.1007/s0016 7-019-05585-1

16. Mutlu H, Sildiroglu H, Pekkafali $\mathrm{Z}$ et al (2006) MRI appearance of retrocalcaneal bursitis and rheumatoid nodule in a patient with rheumatoid arthritis. Clin Rheumatol 25:734-736. https://doi. org/10.1007/s10067-005-0044-x

17. Neuschwander TB, Indresano AA, Hughes TH, Smith BW (2013) Footprint of the lateral ligament complex of the ankle. Foot Ankle Int 34:582-586. https://doi.org/10.1177/1071100712466851

18. Olewnik Ł, Wysiadecki G, Polguj M, Topol M (2017) Anatomic study suggests that the morphology of the plantaris tendon may be related to Achilles tendonitis. Surg Radiol Anat 39:69-75. https:// doi.org/10.1007/s00276-016-1682-1

19. Pastore D, Cerri GG, Haghighi $P$ et al (2009) Ligaments of the posterior and lateral Talar processes: MRI and MR Arthrography of the ankle and posterior subtalar joint with anatomic and histologic correlation. Am J Roentgenol 192:967-973. https://doi. org/10.2214/AJR.08.1207

20. Peduto AJ, Numkarunarunrote N, Trudell DJ, Resnick DL (2009) Fibulotalocalcaneal ligament: magnetic resonance imaging findings with cadaver correlation. J Comput Assist Tomogr 33:444448. https://doi.org/10.1097/RCT.0b013e318184cd89

21. Peterson DA, Stinson W, Carter J (1993) Bilateral accessory soleus: a report on four patients with partial fasciectomy. Foot Ankle 14:284-288. https://doi.org/10.1177/107110079301400509

22. Pingel J, Petersen MCH, Fredberg U et al (2015) Inflammatory and metabolic alterations of Kager's fat pad in chronic achilles tendinopathy. PLoS ONE 10:1. https://doi.org/10.1371/journ al.pone. 0127811

23. Shaw HM, Vázquez OT, McGonagle D et al (2008) Development of the human achilles tendon enthesis organ. J Anat 213:718-724. https://doi.org/10.1111/j.1469-7580.2008.00997.x

24. Schleip R, Klingler W, Lehmann-Horn F (2005) Active fascial contractility: Fascia may be able to contract in a smooth muscle-like manner and thereby influence musculoskeletal dynamics. Med Hypotheses 65:273-277. https://doi.org/10.1016/j. mehy.2005.03.005

25. Schleip R, Hedley G, Yucesoy CA (2019) Fascial nomenclature: Update on related consensus process. Clin Anat 32:929-933. https ://doi.org/10.1002/ca.23423

26. Stecco C, Cappellari A, Macchi V et al (2014) The paratendineous tissues: an anatomical study of their role in the pathogenesis of tendinopathy. Surg Radiol Anat 36:561-572. https://doi. org/10.1007/s00276-013-1244-8

27. van Sterkenburg MN, Kerkhoffs GMMJ, Kleipool RP, Niek van Dijk C (2011) The plantaris tendon and a potential role in mid-portion Achilles tendinopathy: an observational anatomical study. J Anat 218:336-341. https://doi.org/10.111 1/j.1469-7580.2011.01335.x

28. Theobald P, Bydder G, Dent C et al (2006) The functional anatomy of Kager's fat pad in relation to retrocalcaneal problems and other hindfoot disorders. J Anat 208:91-97. https://doi.org/10.11 11/j.1469-7580.2006.00510.x

29. Vaishya R, Agarwal AK, Azizi AT, Vijay V (2020) Haglund's syndrome: a commonly seen mysterious condition. Cureus 8:1. https://doi.org/10.7759/cureus.820

30. Vega J, Malagelada F, Manzanares Céspedes M-C, Dalmau-Pastor M (2020) The lateral fibulotalocalcaneal ligament complex: an ankle stabilizing isometric structure. Knee Surg Sports Traumatol Arthrosc 28:8-17. https://doi.org/10.1007/s00167-018-5188-8

Publisher's Note Springer Nature remains neutral with regard to jurisdictional claims in published maps and institutional affiliations. 